fed through nasogastric or percutaneous endoscopic gastrostomy tubes, may also be at risk.

Understanding of refeeding syndrome and its treatment is limited among general physicians and surgeons. Many patients at risk of refeeding syndrome are not treated on specialist nutrition units. Measurement of serum phosphate may not be done in patients at risk, and when phosphate is measured the importance of grossly abnormal results may not be recognised. The other barrier is a lack of consensus on treatment. Intravenous phosphate is required as oral supplementation is inadequate. Previously recommended regimens for treatment of severe hypophosphataemia have been developed mainly from experience in treating small numbers of patients in intensive care settings. ${ }^{7}$ Typically regimens recommend multiple infusions based on weight, with frequent monitoring of serum phosphate, but these complex regimens may be impractical on general wards. Ideally a treatment regimen for refeeding syndrome would not require monitoring blood tests more often than daily, would not require adjustment for weight, and would provide an adequate dose of phosphate.

Results of such a regimen have recently been published. ${ }^{8}$ Thirty patients with refeeding syndrome, normal renal function, and a phosphate concentration of less than $0.50 \mathrm{mmol} / \mathrm{l}$ were treated with $50 \mathrm{mmol}$ intravenous phosphate over 24 hours $(500 \mathrm{ml}$ Phosphates Polyfusor, Fresenius Kabi, Warrington, United Kingdom). This treatment was effective; 93\% (28/30) achieved a serum phosphate concentration of $0.50 \mathrm{mmol} / \mathrm{l}$ or more after four days. Importantly, five patients required further phosphate as severe hypophosphataemia recurred after initial correction. The treatment seemed safe; no patient developed renal failure, although three patients developed mild transient hyperphosphataemia and four asymptomatic hypocalcaemia. Importantly, all patients were managed on general wards. Although this study is uncontrolled, it is the largest published series of the treatment of severe hypophosphataemia due to refeeding syndrome.

Treatment of refeeding syndrome can be helped by the input of hospitals' nutrition teams. Dieticians and nutrition nurses can help in identifying malnourished patients at risk of developing refeeding syndrome. When these patients require artificial feeding (enteral or parenteral), this should be started at a reduced calorific rate (25-50\% of estimated requirements) to reduce the risk of refeeding syndrome developing. Serum phos- phate, magnesium, calcium, potassium, urea, and creatinine concentrations should be measured before feeding and repeated daily for four days after feeding is started. When hypophosphataemia occurs it should be corrected in addition to other electrolyte abnormalities, such as hypokalaemia and hypomagnesaemia. Feeding can be continued. If after 24 hours the serum phosphate concentration remains low or falls subsequently then further phosphate should be administered.

No randomised controlled trials of treatment for refeeding syndrome have been performed, and the optimal regime therefore remains to be determined. In addition the exact degree of hypophosphataemia requiring treatment remains to be determined, although most experts on nutrition would recommend treatment of hypophosphataemia with measurements of 0.32-0.50 mmol. Randomised controlled trials of the treatment of refeeding syndrome, with clinical end points such as survival, are therefore needed. The current priority is to improve awareness of refeeding syndrome among general physicians and surgeons and to convince them of its importance. Many doctors remain unconvinced of the importance of treating hypophosphataemia, while in contrast accepting the need to treat other electrolyte deficiencies such as hypokalaemia and hypomagnesaemia. With further liaison between hospital nutrition teams and ward staff and the use of an adequate treatment regimen, it may be possible to improve the prognosis of this currently underecognised and undertreated group substantially.

Stephen D Hearing consultant gastroenterologist

Department of Gastroenterology, Staffordshire General Hospital, Weston Road, Stafford ST16 3SA

(stephen.hearing@msgh-tr.wmids.nhs.uk)

Competing interests: None declared

1 Schnitker MA, Mattman PE, Bliss TL. A clinical study of malnutrition in Japanese prisoners of war. Ann Intern Med 1951;35:69-96.

Crook MA, Hally V, Panteli JV. The importance of the refeeding syndrome. Nutrition 2001;17:632-7.

Crook M, Swaminathan R. Disorders of plasma phosphate and indications for its measurement. Ann Clin Biochem 1996;33:376-96.

4 Silvis SE, Paragas PD Jr. Paraesthesias, weakness, seizures, and hypophosphataemia in patients receiving hyperalimentation. Gastroenterolog 1972;62:513-20.

5 Weinster RL, Krumdieck CL. Death resulting from overzealous total parenteral nutrition: The refeeding syndrome revisited. Am J Clin Nutr 1980;34:393-9.

6 Cumming AD, Farquhar JR, Bouchier IAD. Refeeding hypophosphataemia in anorexia nervosa and alcoholism. BMJ 1987;295:490-1.

Perreault MM, Ostrop NJ, Tierney MG. Efficacy and safety of intravenous phosphate replacement in critically ill patients. Crit Care 1997;31:683-8. 8 Terlevich A, Hearing SD, Woltersdorf WW, Smyth C, Reid D, McCullagh E, et al. Refeeding syndrome: effective and safe treatment with phosphates polyfusor. Aliment Pharmacol Ther 2003;17:1325-9.

\title{
Pre-eclampsia and the risk of cancer
}

\section{Several studies suggest a decreased risk-except this one}

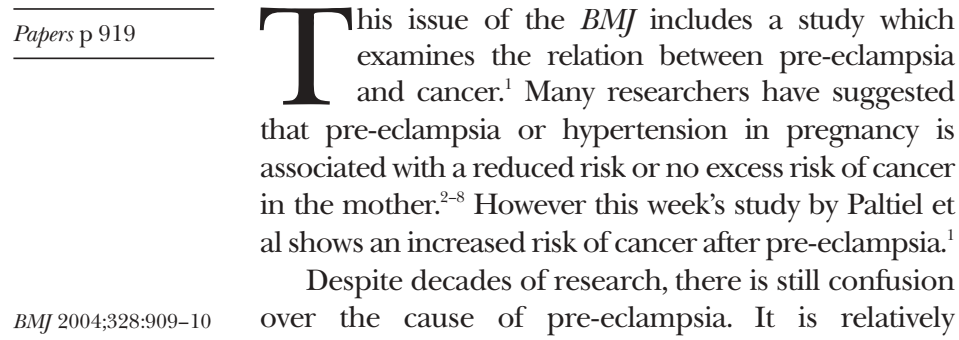

common, affecting $3-5 \%$ of pregnancies, and is a leading cause of morbidity and mortality both in the mother and unborn child. ${ }^{9}$ Several observational studies in European and North American populations have examined the relation between pre-eclampsia (or hypertension) in pregnancy and cancer. ${ }^{2-8}$ Unfortunately some were based on a small number of cancers in the pre-eclampsia group, not all adjusted for known confounders, and the length of follow up varied. Most 
of these studies found a reduced risk of breast cancer after pre-eclampsia or hypertension in pregnancy, ${ }^{2-4} 67$ and one study found no significant association. ${ }^{8}$ Some authors have hypothesised that the observed reduction in risk of maternal breast cancer may be due to reduced maternal oestrogen, increased $\alpha$ fetoprotein, or raised androgen concentrations. ${ }^{3}{ }^{12} 13$ One cohort study found a decreased risk of death from all cancers in the mother, and this risk was further decreased when pre-eclampsia developed before term. The authors hypothesised that this reduction was due to the lower prevalence of smoking and reduced oestrogen levels in mothers with pre-eclampsia. ${ }^{5}$

Studies investigating the relation between preeclampsia (or hypertension) in pregnancy and breast cancer that also provide risk estimates are shown in a forest plot (figure). Although we need to be cautious in interpreting results from case-control and cohort studies together, the overall picture is useful as it illustrates that the results of the present study clearly contrast with previous studies. Wherever possible, the risks shown are those corrected for known confounders and are for women followed up from first pregnancy. The main problems in interpreting any observational study relate to the potential for residual confounding and bias. Many established and probable risk factors for breast cancer exist, ${ }^{14}$ and it is important to correct for as many of these confounders as possible when interpreting the results of such studies.

Paltiel et al present the results of a cohort study of pre-eclampsia with analysis of results restricted to three large hospitals in West Jerusalem where recording of pre-eclampsia was complete (37033 women). ${ }^{1}$ The median follow up was 29 years. The incidence of cancer increased marginally after preeclampsia (hazard ratio 1.27 (95\% confidence interval 1.03 to 1.57). Site-specific increases were noted for cancers of the breast, stomach, lung/larynx, and ovary. The hazard ratio for breast cancer after pre-eclampsia was 1.38 (1.0 to 1.89), adjusted for age at baseline and parity. In women followed up from first birth $(n=22716)$, there was a further increase in age adjusted risk of breast cancer (hazard ratio1.75 (1.19 to 2.58)).

The authors suggest their results may be partly explained by the longer period of follow up in their study. Previous cohort studies have been based on shorter periods (median 13 years, ${ }^{5}$ mean 16 years ${ }^{7}$ and mean 19 years $\left.^{6}\right)$. It would be helpful if the authors could calculate hazard ratios by decade of follow up, to see if this changes significantly with increasing length of follow up.

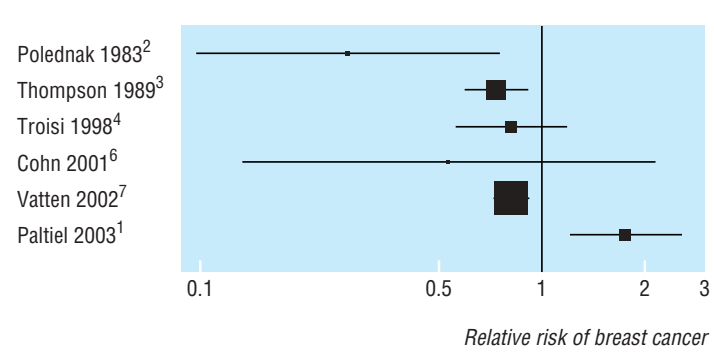

Forest plot of studies examining the association between pre-eclampsia or hypertension in pregnancy and breast cancer

\section{Pre-eclampsia ${ }^{9-11}$}

Definition - raised blood pressure and proteinuria

Pathophysiology-intense vasospasm; activation of coagulation cascade; plasma volume contraction; organ hypoperfusion

Pre-disposing factors-first pregnancy, previous pregnancy with pre-eclampsia, hypertension, diabetes, increased insulin resistance, black race, increased testosterone, genetics, pregnancy with new father, high maternal weight

It is possible that different risk factors for pre-eclampsia exist among different populations, and that, as Paltiel et al propose, ${ }^{1}$ certain populations are at increased risk of cancer after pre-eclampsia. Certain families are more at risk of cancer and, in particular, some genes confer a greatly increased risk of breast cancer. BRAC 1 and 2 genes are more common in Ashkenazi Jewish populations than in Western populations, though only about 2\% of Ashkenazi Jewish women carry these genes. ${ }^{14}$

The available evidence therefore suggests that women in Europe and North America probably have a reduced risk of cancer after pre-eclampsia, at least in the short term. With increasing time, this risk might reverse. It is also possible that women from the Middle East have some common underlying risk factors for certain cancers and pre-eclampsia, possibly related to genetics or lifestyle factors. Unfortunately any explanation for these results is speculative, so further good quality population based studies with long periods of follow up are necessary.

Aliki Taylor Cancer Research UK training fellow in cancer public health and epidemiology

Department of Public Health and Epidemiology, University of Birmingham, Birmingham B15 2TT (A.J.Taylor@bham.ac.uk)

Competing interests: none declared.

1 Paltiel O, Friedlander Y, Tiram E, Barchana M, Xue X, Harlap S. Cancer after pre-eclampsia: follow up of the Jerusalem perinatal study cohort.
$B M J$ 2004;328:919-21. BM) 2004,328:919-21.

2 Polednak AP, Janerich DT. Characteristics of first pregnancy in relation to early breast cancer. A case-control study.J Reprod Med 1983;28:314-8.

Thompson WD, Jacobson HI, Negrini B, Janerich DT. Hypertension, pregnancy and risk of breast cancer. J Natl Cancer Inst 1989;81:1571-4.

4 Troisi R, Weiss HA, Hoover RN, Potischman N, Swanson CA, Brogan DR, et al. Pregnancy characteristics and maternal risk of breast cancer. Epidemiology 1998;9:641-7.

5 Irgens H, Reisaeter L, Irgens LM, Lie RT. Long term mortality of mothers and fathers after pre-eclampsia: population based cohort study. BMJ 2001;323:1213-7.

6 Cohn BA, Cirillo PM, Christianson RE, van den Berg BJ, Siiteri PK. Placental characteristics and reduced risk of maternal breast cancer. J Natl Cancer Inst 2001;93:1133-40.

7 Vatten LJ, Romundstad PR, Trichopoulos D, Skjaervan R. Pre-eclampsia in pregnancy and subsequent risk for breast cancer. $\mathrm{Br} J$ Cancer 2002;87:971-3.

8 Mogren I, Stenlund H, Högberg U. Long-term impact of reproductive factors on the risk of cervical, endometrial, ovarian and breast cancer. Acta Oncologica 2001;40:849-54.

9 Roberts JM, Cooper DW. Pathogenesis and genetics of pre-eclampsia. Lancet 2001;357:53-6.

10 Odegard RA, Vatten LJ, Nielson ST, Salvesen KA, Austgulen R. Risk factors and clinical manifestations of pre-eclampsia. Br J Obstet Gynaecol 2000;107:1410-6.

11 Lie, RT, Rasmussen S, Brunborg H, Gjessing HK, Lie-Nielsen E, Irgens LM. Fetal and maternal contributions to risk of pre-eclampsia: population based study. BMJ 1998;316:1343-7.

12 Innes KE and Byers TE. Preeclampsia and breast cancer risk. Epidemiology 1999;10:722-3.

13 Troisi R, Potischman N, Roberts JM, Ness R, Crombleholme W, Lykins D et al. Maternal serum oestrogen and androgen concentrations in preeclamptic and uncomplicated pregnancies. Int I Epidemiol 2003;32:455-60.

14 McPherson K, Steel CM, Dixon JM. ABC of breast diseases: Breast cancer - epidemiology, risk factors, and genetics. BMJ 2000;321:624-8. 